\title{
Linfedema asociado al cáncer de mama: factores de riesgo, diagnóstico y tratamiento quirúrgico
}

\author{
Nicolás Pereira C. ${ }^{1,2}$, Gemma Pons P. ${ }^{3}$ y Jaume Masià A. ${ }^{3}$
}

\section{Breast cancer related lymphedema: risk factors, diagnosis and surgical treatment}

Breast cancer related lymphedema is one of the most underestimated and debilitating complications of the treatment of this entity. Occurs as result of the interruption of the lymphatic flow in association with other factors. The incidence varies depending on the type of treatment received; being a higher risk in cases in which total mastectomy, axillary dissection, radiotherapy are performed; and in patients in whom the lymph nodes are positive for cancer, there was a greater number of harvested lymph nodes, taxanes were used or in obese patients. Clinical diagnosis and imaging techniques are essential to assess the functional status of the lymphatic system. The main objectives in the management of lymphedema are to limit patient morbidity, improve functionality and quality of life. There are procedures that seek to prevent the development of breast cancer related lymphedema. Once established, the treatment can be conservative and surgical. Surgical treatment includes physiological (reconstructive) and excisional procedures. Success depends on a good selection of patients and the performance of an individualized treatment. The following is a review regarding the incidence, risk factors, diagnostic strategies and surgical techniques with emphasis on microsurgical treatment.

Key words: lymphedema; breast cancer; supermicrosurgery; lymphovenous anastomosis; vascularized lymph-node transfer.

\section{Resumen}

El linfedema asociado al cáncer de mama es una de las complicaciones más subestimadas y debilitantes del tratamiento de esta entidad. Ocurre como resultado de la interrupción del flujo linfático en asociación a otros factores. La incidencia varía dependiendo del tipo tratamiento recibido, existiendo mayor riesgo en los casos en los que se realiza mastectomía total, disección axilar, radioterapia, y en los pacientes en los cuales los ganglios están positivos para cáncer, hubo una mayor cantidad de linfonodos resecados, se utilizaron taxanos o padecen de obesidad. El diagnóstico clínico y a través de técnicas de imágenes es fundamental para evaluar el estado funcional del sistema linfático. Los objetivos principales en el manejo del linfedema son limitar la morbilidad del paciente, mejorar la funcionalidad y la calidad de vida. Existen procedimientos que buscan prevenir el desarrollo del linfedema asociado al cáncer de mama. Una vez establecido, el tratamiento puede ser conservador y quirúrgico. El tratamiento quirúrgico incluye procedimientos fisiológicos (reconstructivos) y resectivos. El éxito depende de una buena selección de los pacientes y la realización de un tratamiento individualizado. A continuación, se presenta una revisión en cuanto a la incidencia, factores de riesgo, estrategias diagnósticas y técnicas quirúrgicas con énfasis en el tratamiento microquirúrgico.

Palabras clave: linfedema; cáncer de mama; supermicrocirugía; anastomosis linfático-venosa; transferencia de linfonodos vascularizada.
Departamento de Cirugía Plástica, Clínica Las Condes. Santiago, Chile.

²Departamento de Cirugía Plástica y Quemados, Hospital del Trabajador. Santiago, Chile.

${ }^{3}$ Departamento de Cirugía Plástica, Hospital de la Santa Creu y Sant Pau. Barcelona, España.

Recibido el 28 de diciembre de 2017 y aceptado para publicación el 11 de mayo de 2018.

Correspondencia a: Dr. Nicolás Pereira C. npereira@clc.cl 


\section{Introducción}

El linfedema asociado al cáncer de mama (BCRL: breast cancer related lymphedema) es una de las complicaciones más subestimadas y debilitantes del tratamiento de esta entidad. La incidencia de BCRL varía entre 3\%-65\% dependiendo del tipo de tratamiento y el tiempo de seguimiento. Una revisión sistemática mostró una incidencia general de $21,4 \%$ en estudios prospectivos, siendo cuatro veces mayor en pacientes con disección axilar $(19,9 \%)$ comparados con biopsia de ganglio centinela $(5,6 \%)^{1}$. Tiene un impacto negativo en la calidad de vida general y representa una carga económica importante para los pacientes, los cuidadores y la sociedad $^{2}$.

El linfedema asociado al cáncer de mama ocurre como resultado de la interrupción del flujo linfático en asociación a otros factores. En el $75 \%$ de los casos se presenta dentro del primer año y en el $90 \%$ dentro de los 3 años posterior de la cirugía ${ }^{3}$.

Un adecuado seguimiento de pacientes con alto riesgo de desarrollar BCRL facilita la implementación precoz de estrategias para reducir el riesgo mediante una vigilancia proactiva y tratamiento, lo cual mejoran los resultados y la calidad de vida.

\section{Factores de riesgo (Tabla 1)}

Con respecto al tratamiento del cáncer de mama, diversos estudios han demostrado que la mastectomía tiene mayor riesgo a desarrollar BCRL que una tumorectomía $(20 \%$ vs $8 \%)(\mathrm{RR}=1,42)^{4}$. Los pacientes sin disección axilar tienen menor riesgo que en los que se realiza la disección $(\mathrm{RR}=3,47)$, y a su vez, la disección axilar aumenta el riesgo en comparación a la biopsia de ganglio centinela $(\mathrm{RR}=3,07)^{1}$. El riesgo es mayor cuando los linfonodos son positivos $(\mathrm{RR}=1,54)$ y mientras más

Tabla 1. Factores de riesgo asociados a BCRL

\begin{tabular}{|l|}
\hline Factores de riesgo \\
\hline Mastectomía total \\
\hline Disección axilar \\
\hline Linfonodos positivos \\
\hline Cantidad de linfonodos resecados \\
\hline Radioterapia \\
Uso de taxanos \\
Obesidad \\
\hline
\end{tabular}

sean los linfonodos resecados, aunque no existe un punto de corte. Adicionalmente, la radioterapia axilar aumenta las tasas de BCRL $(\mathrm{RR}=1,92)^{5}$. Por otro lado, el uso de taxanos durante la quimioterapia se asocia a un mayor riesgo de linfedema transitorio y persistente ${ }^{5}$. Existen nomogramas que permiten predecir la probabilidad de desarrollar linfedema secundario al tratamiento del cáncer de mama a 5 años plazo, basado en la presencia de diferentes factores de riesgo ${ }^{6}$.

El sobrepeso y la obesidad son factores de riesgos establecidos ${ }^{4,5}$. Una paciente que presenta IMC $>30 \mathrm{~kg} / \mathrm{m}^{2}$ tiene mayor probabilidad de presentar BCRL comparado con una de IMC $<25 \mathrm{~kg} / \mathrm{m}^{2}$ (Odds ratio $=2,93$ ). A su vez, la disminución tanto del peso como del IMC reduce significativamente la severidad del linfedema en las pacientes que ya lo padecen?.

El efecto del ejercicio en pacientes con riesgo o con linfedema diagnosticado ha sido controversial. Limitar la actividad puede afectar negativamente en la calidad de vida y puede llevar a la pérdida del trabajo en pacientes cuya ocupación implique actividad física. Es más, el retorno a las actividades previas al tratamiento del cáncer de mama mejoran la función física y el bienestar psicológico. Como ha sido demostrado por varios estudios randomizados, ni el ejercicio aeróbico ni el de resistencia empeoran los síntomas de linfedema. Más aún, mejoran la autoestima, la condición física y composición corporal ${ }^{8}$.

Clásicamente se consideraba que los viajes en avión, vías venosas periféricas y la toma de la presión arterial en la extremidad afectada, podían desencadenar o empeorar la evolución del linfedema secundario al cáncer de mama. Actualmente, no hay evidencia suficiente que apoye el beneficio de prevenir estas prácticas, sin embargo, la recomendación de evitar estas conductas debe ser personalizada 9 .

\section{Diagnóstico}

El diagnóstico del linfedema es un desafío, especialmente, en etapas tempranas (etapa 0 o I). Se recomienda la evaluación preoperatoria y seguimiento posoperatorio de la extremidad ipsilateral y contralateral a intervalos de tiempo estandarizados, sin embargo, no existe una recomendación específica sobre una técnica en particular como gold standard.

Las medidas antropométricas ideales deben ser simples de aplicar, no invasivas, higiénicas, costo efectivas, confiables, reproducibles y cuantificables. La medición volumétrica de la extremidad es lo principal para diagnóstico y el seguimiento de 
la progresión. Si bien, existen numerosas formas disponibles para cuantificar el linfedema (desplazamiento de agua, mediciones circunferenciales, perímetro y espectrometría de bioimpedancia), no hay un método universalmente aceptado.

La medición de la circunferencia se debe realizar con una cinta métrica no extensible y en diferentes puntos de la extremidad. Un aumento de 2 centímetros en la circunferencia en el lado enfermo con respecto al lado sano se usa comúnmente para hacer diagnóstico ${ }^{10}$. Cuando se obtienen múltiples medidas, se puede calcular el volumen utilizando la fórmula del cono trunco (frustrum) ${ }^{11}$.

Desde el punto de vista clínico, la presencia de edema, la sensación de pesadez y adormecimiento de la extremidad en el último año son síntomas predictivos para linfedema. La combinación de estos síntomas con la medición del volumen es la mejor forma de evaluar e identificar cambios asociados BCRL.

Existe un creciente interés en detectar el linfedema en etapas subclínicas o iniciales (cambios de volumen relativo de 5\%-10\%), debido a que un diagnóstico temprano permite una intervención y tratamiento precoz obteniendo mejores resultados. Una vez hecho el diagnóstico clínico y la etapificación según la Sociedad Internacional de Linfología $(0-\mathrm{IV})^{12}$, se deben realizar estudios complementarios para determinar la extensión de la enfermedad y la funcionalidad del sistema linfático ${ }^{13}$.

La linfocintigrafía isotópica es el estudio más común y durante mucho tiempo ha sido considerado gold standard para el diagnóstico de linfedema. Entrega una evaluación estática y dinámica del flujo linfático, evidenciando zonas de bloqueo y/o flujo reverso. El hallazgo linfocintigráfico de vasos linfáticos principales sin colaterales permite predecir una buena respuesta al tratamiento conservador con terapia descongestiva compleja en etapas iniciales ${ }^{14}$.

La linforresonancia magnética (MRL) es capaz de mostrar cambios anatómicos detallados en los vasos linfáticos y linfonodos. Proporciona información individualizada sobre el status funcional del transporte de linfa en los vasos linfáticos y en linfonodos mediante la visualización en tiempo real del flujo linfático contrastado en los canales linfáticos y dentro de los linfonodos ${ }^{15}$.

La linfografía con verde indocianina (ICG) se realiza mediante una inyección subdérmica de ICG en los espacios interdigitales de la mano y observando mediante una cámara de fluorescencia cercana al infrarrojo (ej. SPY ${ }^{\circledR}$, PDE-neo ${ }^{\circledR}$ ). Permite evaluar el sistema linfático en tiempo real, identificando la presencia y ubicación de vasos linfáticos permeables.
Es preciso para estadificar el linfedema según su funcionalidad en patrón lineal (funcional) y patrón de dermal backflow (splash, stardust, difuso) ${ }^{16}$, así como orientar en la indicación del manejo quirúrgi$\mathrm{co}^{17}$ (Figura 1). Se ha reportado su utilidad para la detección precoz de disfunción linfática secundaria al manejo del cáncer de mama y para su oportuno tratamiento, previo a la aparición del edema, teniendo mejor rendimiento que la linfocintigrafía ${ }^{18}$.

\section{Tratamiento}

El tratamiento del BCRL debe estar a cargo de un equipo multidisciplinario. Los objetivos principales del manejo del BCRL son limitar la morbilidad de la paciente, mejorar la funcionalidad y la calidad de vida. Con el fin de lograr esto, se debe disminuir el edema, mejorar la higiene, reducir las tasas de infección, mejorar la movilidad y disminuir la carga de las terapias diarias. Por lo tanto, estos factores se deben tener en cuenta al momento de evaluar los resultados del tratamiento.

Existen procedimientos que buscan prevenir el desarrollo del linfedema. Una vez establecido, el tratamiento puede ser conservador o quirúrgico. El tratamiento quirúrgico, a su vez, incluye procedimientos fisiológicos (reconstructivos) y resectivos.

\section{Tratamiento preventivo}

La primera medida preventiva del linfedema es llevar a cabo una técnica quirúrgica extremadamente cuidadosa al practicar la biopsia de ganglio centinela o disección axilar.

La técnica de mapeo axilar reverso para prevenir el linfedema consiste en el mapeo linfático de la extremidad superior con azul patente y el de la

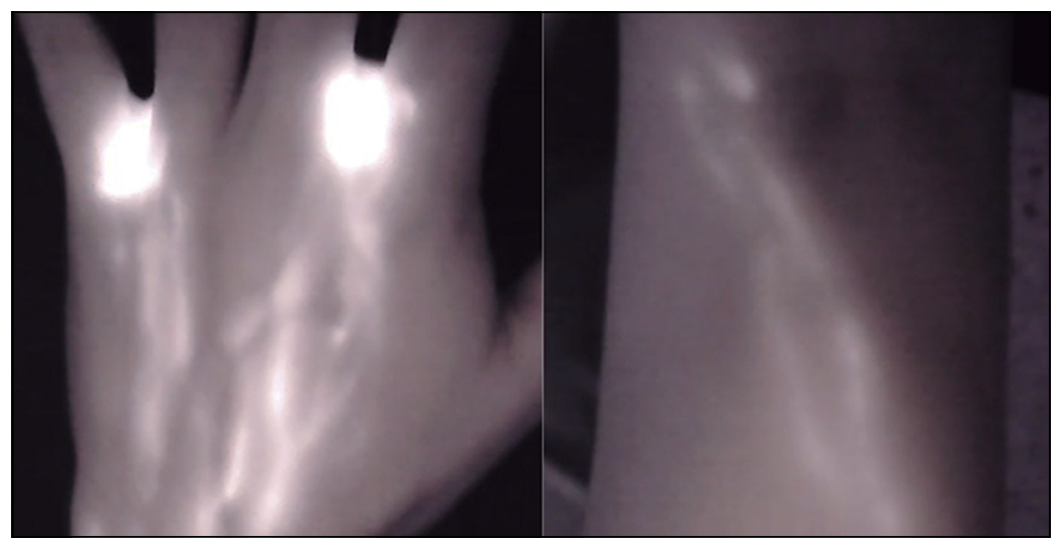

Figura 1. Linfografía con ICG en un paciente con linfedema de extremidad superior asociado a cáncer de mama en donde se observa un patrón lineal (funcional). 
mama con Tecnecio-99, al momento que se realiza la biopsia de ganglio centinela o disección axilar. Permite diferenciar los linfáticos que drenan la mama (calientes) de los que drenan el brazo (azules) para evitar dañarlos y, en caso de seccionarlos, realizar anastomosis linfático-linfáticas y disminuir el riesgo de linfedema ${ }^{19}$. Existen variaciones en el patrón de drenaje linfático de la extremidad superior, pudiendo coexistir o cruzarse con el de la mama. Utilizando el del mapeo axilar reverso, se presentó linfedema en el 0,8\% de las biopsia de ganglio centinela y $6,5 \%$ de las disecciones axilares ${ }^{19}$. En un estudio prospectivo con 20 meses de seguimiento, pacientes con disección axilar presentaron BRCL en un $33 \%$, mientras que en las pacientes que se agregaba el mapeo axilar reverso fue del $6 \%{ }^{20}$.

El abordaje linfático microquirúrgico preventivo curativo (LYMPHA, por sus siglas en inglésLymphedema Microsurgical Preventive Healing Approach) es una técnica quirúrgica de prevención primaria de linfedema luego de la disección axilar. Consiste en la realización de anastomosis linfáticovenosas múltiples al momento de la disección axilar, entre los linfáticos principales seccionados que drenan la extremidad superior y ramas de la vena axilar. En estudios iniciales se utilizó en casos de linfocintigrafía con índice de tránsito mayor a 10 y/o IMC $>30 \mathrm{~kg} / \mathrm{m}^{2}$. En un seguimiento a 4 años de 74 pacientes, sólo un 4\% presentaron linfede$\mathrm{ma}^{21}$; mientras que en otro estudio con 22 meses de seguimiento, sólo un 7\% de los pacientes en que se realizó disección axilar más LYMPHA desarrollaron linfedema comparado con un $31 \%$ en pacientes solamente con disección axilar ${ }^{22}$.

\section{Tratamiento conservador}

Tradicionalmente, el tratamiento conservador es el pilar fundamental y la forma inicial de tratamiento del linfedema. En los casos en etapa 0 (subclínico), se debe realizar un seguimiento combinado con uso discrecional de prendas compresivas como medidas profilácticas. Cuando el linfedema ya es evidente (etapas I, II, III), la terapia descongestiva compleja (TDC) debe ser realizada por un profesional especializado en linfedema, siendo más efectiva en etapas tempranas. Tiene una fase reductiva inicial (3-8 semanas) que consiste en el drenaje linfático manual, vendaje multicapa, ejercicios terapéuticos, compresión elástica, cuidado de la piel, educación y autocuidado; y una fase de mantenimiento que contempla el autodrenaje linfático permanente, ejercicios, cuidado de la piel y uso de prendas/vendaje de compresión ${ }^{12}$.

La TDC, logra un 40\%-60\% de reducción del ex- ceso de volumen en pacientes con edema y fóvea ${ }^{23}$. Si bien son efectivas, estas terapias tienen sus detractores; no solamente debido a que el tratamiento debe ser indefinido para mantener su eficacia, sino porque existe una amplia variabilidad en la calidad de los tratamientos y de las prendas compresivas.

Como se mencionó previamente, las medidas que previenen la progresión del linfedema con evidencia científica son mantener el peso normal, evitar la ganancia de peso y participar en programas de ejercicios supervisados.

\section{Tratamiento quirúrgico}

\section{Procedimientos fisiológicos o reconstructivos}

Actualmente, los procedimientos fisiológicos más comúnmente realizados en el linfedema secundario al cáncer de mama son las anastomosis linfático-venosas y la transferencia de linfonodos vascularizados.

\section{Anastomosis linfático-venosas (LVA)}

Es el procedimiento fisiológico más frecuente y consiste en crear derivaciones linfático-venosas en la extremidad afectada usando conductos linfáticos distal a la obstrucción para redirigir el flujo linfático a la circulación venosa. La gradiente de presión linfático-venosa y la presencia de válvulas competentes en las venas permite el flujo linfático a través de la anastomosis. Se puede realizar proximal o distal en la extremidad, pero en esta última la presión venosa podría impedir el flujo de linfa a través de ésta. La reducción del exceso de volumen varía con cada técnica y depende de la etapa de la enfermedad.

El procedimiento consiste en la inyección subdérmica de ICG en los espacios interdigitales de la mano; mediante una cámara cercana al infrarrojo marcar los vasos linfáticos funcionales y correlacionarlo con los hallazgos de la MRL, en caso de disponer de ella. Se realiza una incisión transversal para identificar y aislar un vaso linfático y su vénula adyacente. Se realiza un bypass (LVA) términoterminal o latero-terminal (Figura 2).

No hay consenso respecto al momento de la cirugía, el número, localización y configuración de las LVA, pero se acepta que múltiples LVA proximales pueden tratar o incluso curar un BCRL en etapa inicial, permitiendo a algunos pacientes discontinuar el uso de prendas compresivas y la fisioterapia luego de unos meses tras la cirugía. Esto se debe a la presencia de linfáticos funcionantes y a un mínimo depósito de tejido fibroadiposo.

Diversos estudios han demostrado la eficacia de 
la LVA en el tratamiento del linfedema. Muchos de estos reportes incluyen un limitado número de pacientes, pero demuestran una reducción aproximada del $35 \%$ al $50 \%$ de la circunferencia o el volumen, en seguimientos mayores a 1 año ${ }^{24}$. Chang et al. reportó un análisis prospectivo de LVA en 89 pacientes consecutivos con 12 meses de seguimiento, encontrando una reducción media del volumen del $61 \%$ en las primeras etapas del BCRL y del $17 \%$ en estadio avanzado de linfedema ${ }^{25}$. Cabe destacar que la mejoría más importante se observa en la etapa temprana del linfedema de extremidad superior, con una meseta después de 1 año. Otros estudios han corroborado estos hallazgos, complementando a la disminución del volumen, una disminución de las tasas de infección y la suspensión de las prendas de compresión después de $\mathrm{LVA}^{26}$. Las complicaciones de la LVA son inusuales y mínima, incluyendo infección, fístula linfática y problemas de cicatrización de la herida. Los beneficios de la técnica son lo mínimamente invasivo, escaso dolor y posibilidad de alta a las 24-48 horas.

\section{Transferencia de linfonodos vascularizados (VLNT)}

Descrito inicialmente por Becker et al. para el tratamiento del linfedema ${ }^{27}$, es un procedimiento microquirúrgico que consiste en la transferencia de un colgajo libre de linfonodos a la extremidad afectada por BRCL, ya sea a la axila (ortotópico) o distal (heterotópico).

Las teorías que explican el mecanismo de acción de VLNT son que los linfonodos trasferidos actuarían como una "esponja o bomba" que atrae linfa y pasa al sistema venoso mediante conexiones linfático-venosas naturales dentro del colgajo ${ }^{28} \mathrm{y} / \mathrm{o}$ a través de linfoangiogénesis en el lecho receptor mediada por factor de crecimiento vascular endotelial C (VEGF-C) que estimula el crecimiento e inosculación de vasos linfáticos a la red linfática de los $\mathrm{VLNT}^{29}$.

Para el tratamiento de BCRL, los linfonodos más frecuentemente utilizados para VLNT son los inguinales superficiales ${ }^{30,31}$. Otras zonas dadoras incluyen los supraclaviculares, submentales, torácicos y gastroepiploicos ${ }^{1,32}$.

Cuando se utilizan los linfonodos inguinales o torácicos (axilares) existe un riesgo potencial de linfedema iatrogénico de la zona dadora. El "mapeo linfático reverso" permite diferenciar los linfonodos que drenan la extremidad inferior (inguinales) o superior (torácicos) con un radionucleótido marcado, de los linfonodos que drenan el abdomen inferior o el tórax con ICG, respectivamente ${ }^{33}$. Aunque el

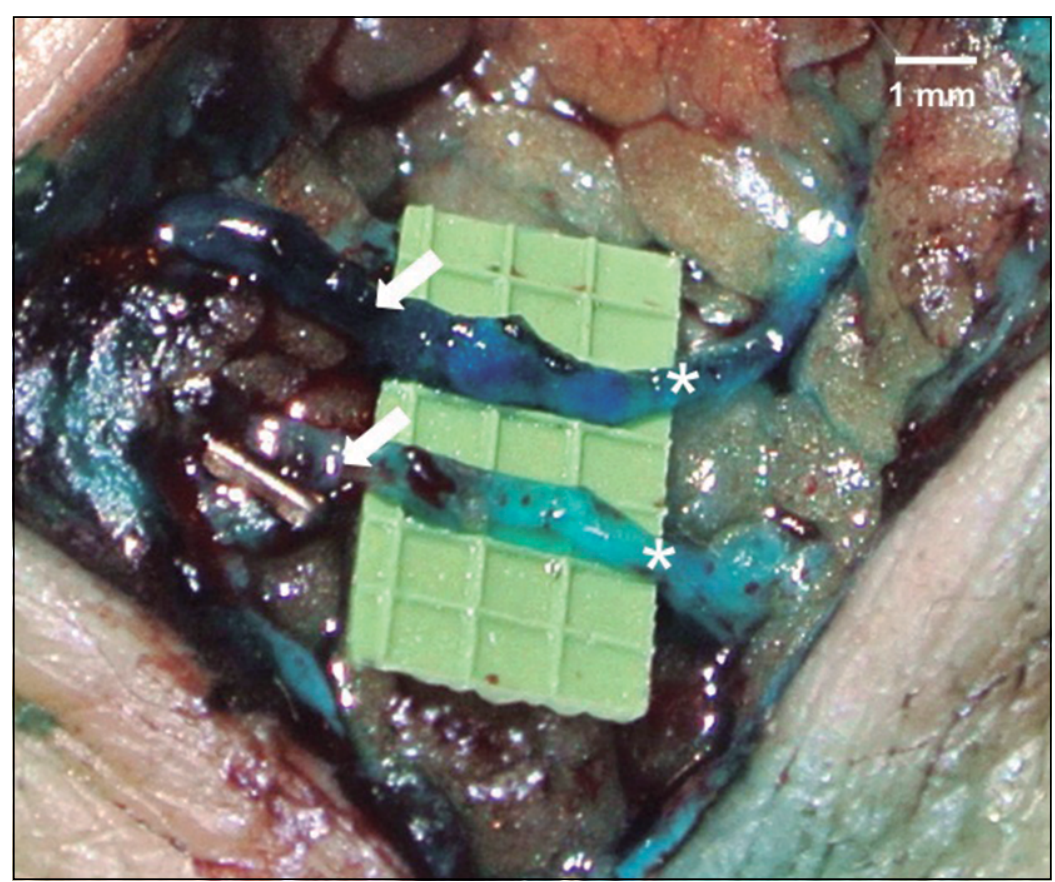

Figura 2. Anastomosis linfático-venosa para el tratamiento del BCRL con técnica de supermicrocirugía (vasos de 0,8 mm). Asteriscos, venas; flechas, vasos linfáticos.

riesgo de linfedema iatrogénico de la zona dadora luego de VLNT es bajo, es un riesgo real y debe ser discutido con el paciente.

Las zonas receptoras descritas para el BCRL son axila, codo y muñeca. Cuando se propone la axila (ortotópico) luego de una cirugía previa en la zona, se debe remover todo el tejido cicatricial hasta plano vital y reemplazarlo por un colgajo bien vascularizado $^{32}$. Además, los colgajos en esta ubicación se pueden ocultar de mejor manera y otorgar un mejor resultado estético. Por el contrario, las zonas distales (heterotópicas) como receptora de VLNT son más fáciles de disecar y aportarían el efecto "esponja" como mecanismo de drenaje, por lo que en teoría comienzan a funcionar inmediatamente.

Diversos estudios han reportado reducciones significativas en la circunferencia o el volumen de un $30 \%$ a $60 \%$ luego de $\mathrm{VLNT}^{34}$. En la mayoría de los casos se trata de estudios retrospectivos, sin embargo, Cheng et al. en un trabajo prospectivo en pacientes con BCRL, encontraron una mayor reducción en la circunferencia de 10 pacientes que recibieron VLNT (40\%) comparado con 10 pacientes controles que eligieron tratamiento conservador $(8 \%)$ en un período de seguimiento de 39 meses $^{30}$. Patel et al. reportaron un $24 \%$ de reducción en la circunferencia de la extremidad superior en un estu- 
dio prospectivo de 15 pacientes con seguimiento de 25 meses promedio ${ }^{35}$. Recientemente, Engel et al. reportaron mejores resultados utilizando VLNT, con o sin reconstrucción mamaria simultánea, compararado con LVA y $\mathrm{TDC}^{36}$, en cuanto a la disminución de la circunferencia, tasa de reducción y a episodios de celulitis. Por otro lado, mediante linfocintigrafía se ha demostrado una mejora en el drenaje linfático de los pacientes con VLNT.

La VLNT de linfonodos inguinales se puede realizar en conjunto con la reconstrucción mamaria a través de un colgajo abdominal basado en la arteria perforante de epigástrica inferior profunda (DIEP) o en la arteria epigástrica inferior superficial (SIEA) ${ }^{37}$ (Figura 3). Está indicado en pacientes que requieren reconstrucción mamaria y presentan $\mathrm{BCRL}$ secundario a la disección axilar. Como se mencionó previamente, la resección del tejido cicatricial en la axila es muy importante previo a la transferencia del colgajo de linfonodos, para descomprimir la vena axilar y permitir la linfangiogénesis entre el colgajo y el lecho receptor. El pedículo del colgajo abdominal se anastomosa a los vasos mamarios internos o toracodorsales y los linfonodos transferidos a los vasos toracodorsales (de manera anterógrada o retrógrada) o sus ramas. Se ha descrito una disminución del perímetro y mejoría sintomática en alrededor del 78\%-79\% de los pacientes. Al comparar la VLNT con DIEP+VLNT, se obtienen reducciones del volu-

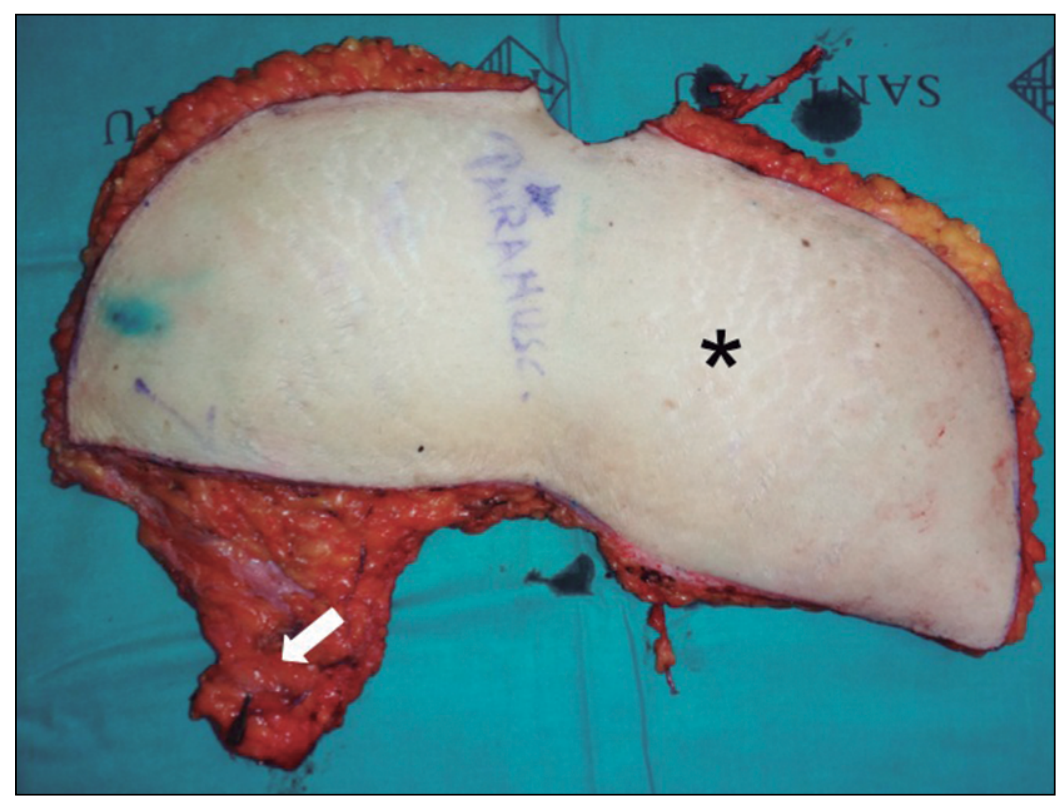

Figura 3. Transferencia de linfonodos vascularizados en conjunto de colgajo DIEP para el tratamiento del BCRL. Asterisco, colgajo DIEP; flecha, linfonodos inguinales. men similares, sin embargo, en la cirugía que combina la reconstrucción mamaria con la transferencia de linfonodos se consigue una mayor mejoría de la función linfática y disminución en la necesidad de terapia compresiva ${ }^{38}$.

\section{Procedimientos resectivos}

Los procedimientos de Charles y reducción radical del linfedema con preservación de perforantes (RRPP), tienen un rol limitado en el manejo del BCRL.

La liposucción puede ser utilizada de manera aislada o en combinación a otros procedimientos para tratar el linfedema. Remueve el tejido graso infiltrado que no responde a TCD o a procedimientos fisiológicos, pero lamentablemente no elimina la necesidad de tratamiento compresivo continuo en el posoperatorio ${ }^{39}$. A pesar de estas deficiencias, la liposucción ha demostrado disminuir el edema, las tasas de infección en la extremidad linfoedematosa, por lo que continúa siendo una herramienta muy útil para el tratamiento del linfedema severo o como técnica complementaria.

\section{Enfrentamiento de la paciente con linfedema asociado al cáncer de mama}

Se debe realizar una evaluación completa de manera de poder ofrecer un tratamiento individualiza$\mathrm{do}^{37,40}$ (Figura 4). El tratamiento se basa en la etapa clínica, consideraciones anatómicas/funcionales, necesidad de reconstruir la mama y el deseo de la paciente. Los casos que presentan edema con fóvea y respuesta parcial al tratamiento conservador, son candidatas para procedimientos fisiológicos ya que el edema se debe, principalmente, a la acumulación de fluido linfático que puede ser resuelto mediante LVA o VLNT. En casos más avanzados, el aumento de volumen se debe a la acumulación de tejido adiposo y fibrosis tisular, por lo que son candidatos a liposucción.

Para decidir qué procedimiento fisiológico realizar, primero se debe hacer el diagnóstico con linfocintigrafía y evaluar la funcionalidad del sistema linfático mediante linfografía con ICG y/o MRL. Si el sistema linfático aún es funcional y los linfonodos axilares están intactos, se ofrecen LVA. Si el sistema linfático es funcional, pero los linfonodos axilares fueron resecados en la cirugía del cáncer, lo siguiente es evaluar la necesidad de realizar reconstrucción mamaria. Si necesita reconstrucción y tiene antecedentes de disección axilar, se realiza un procedimiento combinado de colgajo abdominal y 


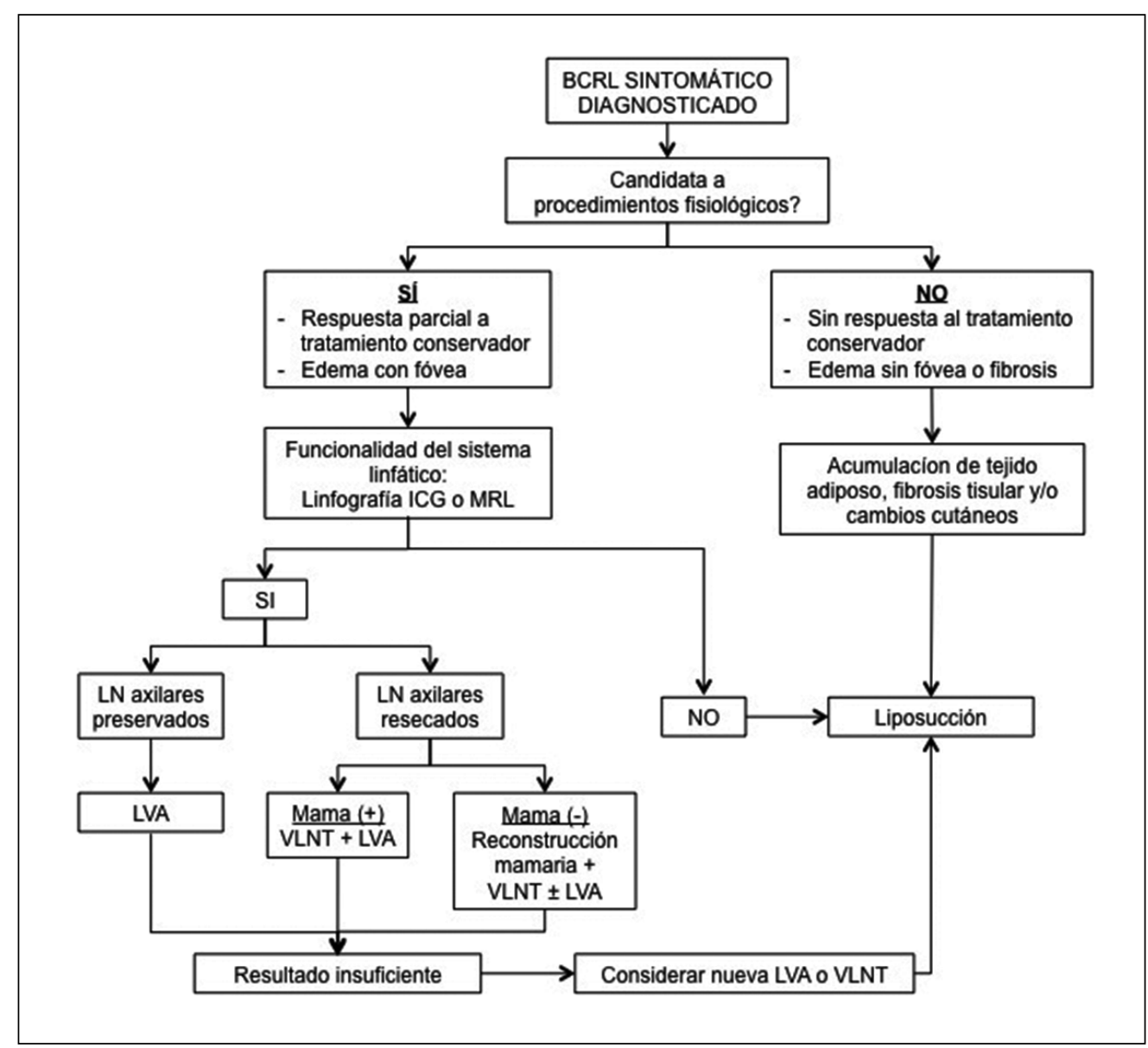

Figura 4. Algoritmo general para el tratamiento de pacientes con linfedema asociado al cáncer de mama. ICG, verde indocianina; MRL, linforresonancia magnética; LN, linfonodos; LVA, anastomosis linfático-venosas; VLNT, transferencia de linfonodos vascularizados.
VLNT \pm LVA asociada; si no necesita reconstrucción y tiene antecedentes de disección axilar, se hace una VLNT+LVA. En caso de no presentar un sistema linfático funcional, la paciente es candidata para un procedimiento reductivo como liposucción ${ }^{37}$.

En los casos en que se realiza procedimientos fisiológicos, durante el período posoperatorio los pacientes deben discontinuar la terapia física y reasumir progresivamente sus actividades normales según tolerancia. El uso de prendas elásticas en este período es controversial. Luego del período posoperatorio inicial, los pacientes deben controlarse periódicamente. Los resultados se evidencian desde los 3 meses y se estabilizan al año posoperatorio. Los resultados se evalúan de acuerdo a la mejoría subjetiva del paciente, medidas perimetrales y volumétricas, frecuencia de episodios de celulitis y cuestionarios de calidad de vida (Figura 5).

\section{Conclusiones}

El linfedema secundario al cáncer de mama es una entidad muy frecuente e invalidante que debe ser manejado por un equipo multidisciplinario. Existen factores de riesgo modificables que deben ser considerados dentro de la terapia. Las nuevas estrategias diagnósticas y las emergentes técnicas microquirúrgicas, tanto para la prevención como para el tratamiento, tienen el potencial de reconstituir fisiológicamente el drenaje linfático alterado. El éxito depende de una buena elección de los pacientes y la realización de un tratamiento individualizado. La comprensión del proceso fisiopatológico, el entendimiento del diagnóstico adecuado, la investigación y el desarrollo de estudios prospectivos nos ayudarán a tratar de mejor manera esta enfermedad y a mejorar la calidad de vida de nuestras pacientes. 


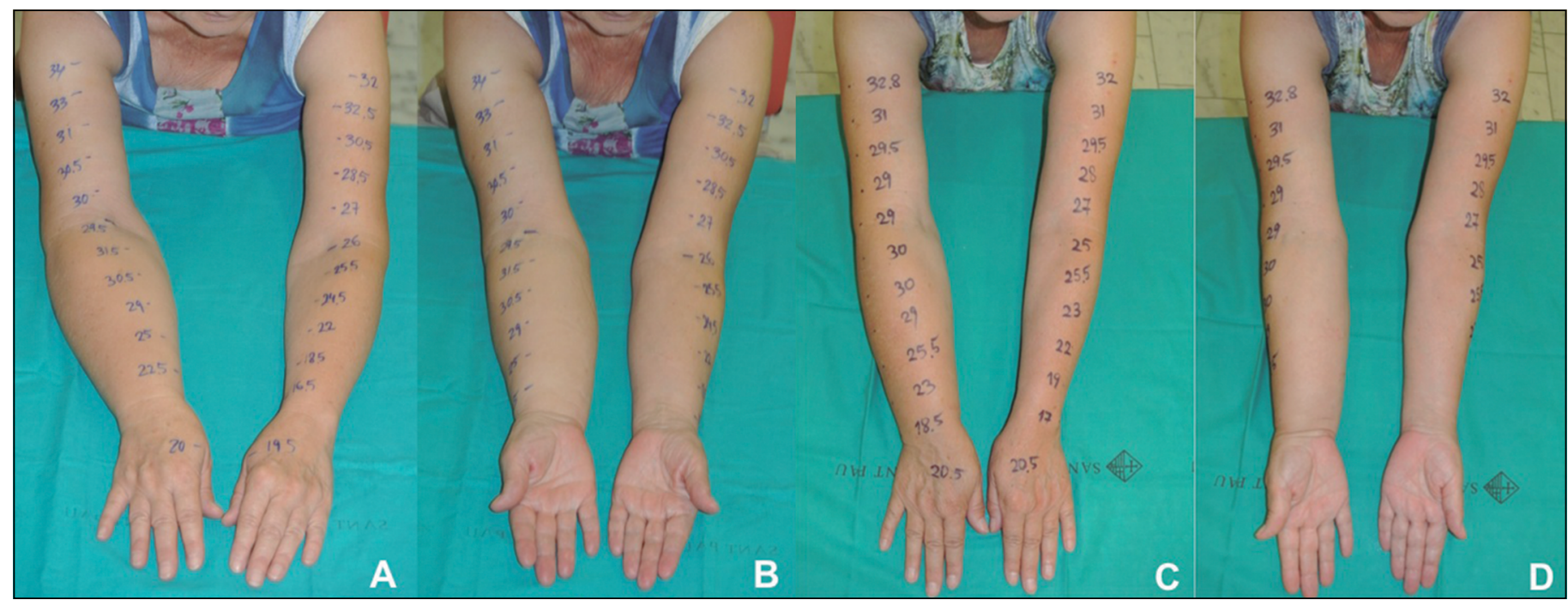

Figura 5. Paciente con $B C R L$ de la extremidad superior derecha antes de las anastomosis linfático-venosas (A-B) y a los 12 meses posoperatorio (C-D).

\section{$\underline{\text { Responsabilidades éticas }}$}

Protección de personas y animales. Los autores declaran que para esta investigación no se han realizado experimentos en seres humanos ni en animales.

Confidencialidad de los datos. Los autores declaran que en este artículo no aparecen datos de pacientes.

Derecho a la privacidad y consentimiento informado. Los autores declaran que en este artículo no aparecen datos de pacientes.

Conflictos de interés: No hay.

\section{Referencias}

1. DiSipio T, Rye S, Newman B, Hayes S. Incidence of unilateral arm lymphoedema after breast cancer: a systematic review and meta-analysis. Lancet Oncol. 2013; 14:500-5.

2. Basta MN, Fox JP, Kanchwala SK, Wu LC, Serletti JM, Kovach SJ, et al. Complicated breast cancer-related lymphedema: evaluating health care resource utilization and associated costs of management. Am J Surg. 2016;211:13341.

3. Rockson SG. Precipitating factors in lymphedema: myths and realities. Cancer 1998;83(12 Suppl American):2814-6.

4. Ozcinar B, Guler SA, Kocaman N, Ozkan M, Gulluoglu BM, Ozmen V. Breast cancer related lymphedema in patients with different loco-regional treatments. Breast 2012;21:361-5.

5. Kilbreath SL, Refshauge KM, Beith JM,
Ward LC, Ung OA, Dylke ES, et al. Risk factors for lymphoedema in women with breast cancer: a large prospective cohort. Breast 2016;28:29-36.

6. Bevilacqua JL, Kattan MW, Changhong Y, Koifman S, Mattos IE, Koifman RJ, et al. Nomograms for predicting the risk of arm lymphedema after axillary dissection in breast cancer. Ann Surg Oncol. 2012;19:2580-9.

7. Shaw C, Mortimer P, Judd PA. A Randomized Controlled Trial of Weight Reduction as a Treatment for Breast Cancer-related Lymphedema. Cancer 2007;110:1868-74.

8. McKenzie DC, Kalda AL. Effect of Upper Extremity Exercise on Secondary Lymphedema in Breast Cancer Patients: A Pilot Study. J Clin Oncol. 2003;21:463-6.

9. McLaughlin SA, DeSnyder SM, Klimberg S, Alatriste M, Boccardo F, Smith ML, et al. Considerations for Clinicians in the Diagnosis, Prevention, and Treatment of Breast Cancer-Related Lymphedema, Recommendations from an Expert Panel: Part 2: Preventive and Therapeutic Options. Ann Surg Oncol. 2017;24:282735.

10. Armer JM, Stewart BR. Post-breast cancer lymphedema: incidence increases from 12 to 30 to 60 months. Lymphology 2010;43:118-27.

11. Taylor R, Jayasinghe UW, Koelmeyer L, Ung O, Boyages J. Reliability and validity of arm volume measurements for assessment of lymphedema. Phys Ther. 2006;86:205-14.

12. International Society of Lymphology. The diagnosis and treatment of peripheral lymphedema: 2013 Consensus Document of the International Society of Lymphology. Lymphology. 2013;46:1-11.

13. Pereira N, Koshima I. Linfedema: Actualización en el Diagnóstico y Tratamiento Quirúrgico. Rev Chil Cir. 2018. (EN PRENSA) 
14. Hwang JH, Choi JY, Lee JY, Hyun SH, Choi Y, Choe YS, et al. Lymphscintigraphy predicts response to complex physical therapy in patients with early stage extremity lymphedema. Lymphology. 2007;40:172-6.

15. Arrivé L, Derhy S, El Mouhadi S, Monnier-Cholley L, Menu Y, Becker C. Noncontrast magnetic resonance lymphography. J Reconstr Microsurg. 2016;32:80-6.

16. Yamamoto T, Yamamoto N, Doi K, Oshima A, Yoshimatsu H, Todokoro $\mathrm{T}$, et al. Indocyanine Green-Enhanced Lymphography for Upper Extremity Lymphedema: A Novel Severity Staging System Using Dermal Backflow Patterns. Plast Reconstr Surg. 2011;128:941-7.

17. Akita S, Mitsukawa N, Kazama T, Kuriyama M, Kubota Y, Omori N, et al. Comparison of lymphoscintigraphy and indocyanine green lymphography for the diagnosis of extremity lymphoedema. J Plast Reconstr Aesthet Surg. 2013;66:7928.

18. Mihara M, Hara H, Araki J, Kikuchi K, Narushima M, Yamamoto T, et al. Indocyanine green (ICG) lymphography is superior to lymphoscintigraphy for diagnostic imaging of early lymphedema of the upper limbs. PLoS One. 2012;7:e38182.

19. Tummel E, Ochoa D, Korourian S, Betzold R, Adkins L, McCarthy M, et al. Does axillary reverse mapping prevent lymphedema after lymphadenectomy? Ann Surg. 2017;265:987-92.

20. Yue T, Zhuang D, Zhou P, Zheng L, Fan Z, Zhu J. A prospective study to assess the feasibility of axillary reverse mapping and evaluate its effect on preventing lymphedema in breast cancer patients. Clin Breast Cancer. 2015;15:301-6.

21. Boccardo F, Casabona F, De Cian F, Friedman D, Murelli F, Puglisi M, et al. Lymphatic microsurgical preventing healing approach (LYMPHA) for primary surgical prevention of breast cancerrelated lymphedema: over 4 years followup. Microsurgery 2014;34:421-4.

22. Gomberawalla A, Borden B, Rohde C, Ascherman J, Taback B, Chen M, et al. Lymphatic microsurgical preventive healing approach (LYMPHA) for the primary prevention of lymphedema. In: Poster presentation 39th Annual San Antonio Breast Cancer Symposium P201-14;2016.

23. Warren AG, Brorson H, Borud LJ, Slavin SA. Lymphedema: a comprehensive review. Ann Plast Surg. 2007;59:464-72

24. Koshima I, Inagawa K, Urushibara K, Moriguchi T. Superrosurgical lymphaticovenular anastomosis for the treatment of lymphedema in the upper extremities. J Reconstr Microsurg. 2000;16:437-42.

25. Chang DW, Suami H, Skoracki R. A prospective analysis of 100 consecutive lymphovenous bypass cases for treatment of extremity lymphedema. Plast Reconstr Surg. 2013;132:1305-14.

26. Damstra RJ, Voesten HG, van Schelven $\mathrm{WD}$, van der Lei B. Lymphatic venous anastomosis (LVA) for treatment of secondary arm lymphedema. A prospective study of 11 LVA procedures in 10 patients with breast cancer related lymphedema and a critical review of the literature. Breast Cancer Res Treat 2009;113:199-206.

27. Becker C, Assouad J, Riquet M, Hidden G. Postmastectomy lymphedema: longterm results following microsurgical lymph node transplantation. Ann Surg. 2006;243:313-5.

28. Cheng MH, Huang JJ, Wu CW, Yang CY, Lin CY, Henry SL, et al. The mechanism of vascularized lymph node transfer for lymphedema: Natural lymphaticovenous drainage. Plast Reconstr Surg. 2014;133:192e-198e.

29. Aschen SZ, Farías-Eisner G, Cuzzone DA, Albano NJ, Ghanta S, Weitman ES, et al. Lymph node transplantation results in spontaneous lymphatic reconnection and restoration of lymphatic flow. Plast Reconstr Surg. 2014;133:301-10.

30. Cheng MH, Chen SC, Henry SL, Tan BK, Lin MC, Huang JJ. Vascularized groin lymph node flap transfer for postmastectomy upper limb lymphedema: Flap anatomy, recipient sites, and outcomes. Plast Reconstr Surg. 2013;131:1286-98.

31. Lin CH, Ali R, Chen SC, Wallace C,
Chang YC, Chen HC, et al. Vascularized groin lymph node transfer using the wrist as a recipient site for management of postmastectomy upper extremity lymphedema. Plast Reconstr Surg. 2009;123:1265-75.

32. Becker C, Vasile JV, Levine JL, Batista BN, Studinger RM, Chen CM, et al. Microlymphatic surgery for the treatment of iatrogenic lymphedema. Clin Plast Surg. 2012;39:385-98.

33. Dayan JH, Dayan E, Smith ML. Reverse lymphatic mapping: A new technique for maximizing safety in vascularized lymph node transfer. Plast Reconstr Surg. 2015; 135:277-85.

34. Raju A, Chang DW. Vascularized lymph node transfer for treatment of lymphedema: A comprehensive literature review. Ann Surg. 2015;261:1013-23.

35. Patel KM, Lin CY, Cheng MH. A prospective evaluation of lymphedemaspecific quality-of-life outcomes following vascularized lymph node transfer. Ann Surg Oncol. 2015;22:2424-30.

36. Engel H, Lin CY, Huang JJ, Cheng MH. Outcomes of Lymphedema Microsurgery for Breast Cancer-related Lymphedema With or Without Microvascular Breast Reconstruction. Ann Surg. 2017 Jun 7. doi: $10.1097 /$ SLA.0000000000002322. [Epub ahead of print].

37. Masià J, Pons G, Rodríguez-Bauzà E. Barcelona Lymphedema Algorithm for Surgical Treatment in Breast CancerRelated Lymphedema. J Reconstr Microsurg. 2016;32:329-35.

38. Akita S, Tokumoto H, Yamaji Y, Sasahara Y, Kubota Y, Kubo M, et al. Contribution of Simultaneous Breast Reconstruction by Deep Inferior Epigastric Artery Perforator Flap to the Efficacy of Vascularized Lymph Node Transfer in Patients with Breast Cancer-Related Lymphedema. J Reconstr Microsurg. 2017;33:571-8.

39. Brorson H. Liposuction in Lymphedema Treatment. J Reconstr Microsurg. 2016;32:56-65.

40. Kung TA, Champaneria MC, Maki JH, Neligan PC. Current Concepts in the Surgical Management of Lymphedema. Plast Reconstr Surg. 2017;139:1003e-1013e. 